\title{
Turbulence modeling with the boundary element method
}

\author{
J. Lupše, L. Škerget \& J. Ravnik \\ University of Maribor, Faculty of Mechanical Engineering, Slovenia
}

\begin{abstract}
In the present article we were testing our flow solver for turbulent channel flow. Velocity-vorticity formulation of Navier-Stokes equations is applied, thus governing equations are given for the kinematic and kinetic aspects of flow instead of mass and momentum equations. The solution algorithm first solves the kinematics equation for unknown boundary vorticity values using the single domain boundary element numerical method. The next step of the solution algorithm is calculation of the domain velocity field, which is also achieved by solving the kinematics equation. In this and later cases we use the sub-domain boundary element method. After the velocity field is known, we calculate the turbulent kinetic energy and turbulent dissipation fields to obtain the turbulent viscosity. Finally, the vorticity field redistribution is calculated via the kinetics equation. For laminar solutions it was shown that the use of the boundary domain integral method accuracy of solutions for benchmark test cases is very high on coarse meshes. However, since this method is still limited, with high CPU and memory requirements, parallelization of the algorithm is a must for calculating turbulent flows. This was achieved with the use of a MPI (message passing interface) standard.
\end{abstract}

\section{Introduction}

In this paper we present the application of the boundary element method for solving planar turbulent flow problems with low-Reynolds-number turbulence models. Since viscosity now includes an additional, modeled part, governing equations have to be written in extended forms, which have to include this nonlinear behavior. The compressibility effect was neglected for simplifying an already highly nonlinear set of equations. For the same reason a zero gravity 
environment was applied. Governing equations are written in velocity-vorticity form, which eliminates pressure from equations that describe incompressible fluid flow. Instead of momentum conservation equations, velocity-vorticity formulation gives the equation for vorticity transport. The mass conservation equation is transformed into a kinematics equation with the help of vorticity definition. Kinematics equations represent compatibility and restriction conditions between velocity and vorticity field functions. These equations are derived from the mass conservation law with the help of vorticity definition. Kinetics equations are obtained by applying a curl differential operator to the momentum transport equation. For application of the turbulence model, the stress tensor has to be rewritten in such a way that we obtain appropriate form for the application of boundary elements. The Laplace fundamental solution was used for kinematics equations integral forms, which are then discretized by the single-domain boundary element method. As this method is very memory consuming, we applied the wavelet transform [1] of domain matrices in order to reduce its size. The resulting system of equations is then solved by direct solver using LU decomposition. The parabolic-diffusion fundamental solution was used in the integral form of kinetics equations, which are then discretized by the sub-domain boundary element method. This type of discretization yields an overdetermined system of equations, which is solved by a LSQR [2] type solver. Obtaining a solution is very challenging due to the strong nonlinearity of the vorticity equation.

\section{Governing equations}

Governing equations describing viscid fluid flow are conservation equations, e.g. mass, momentum and heat energy conservation equations. For isothermal flows, energy conservation equation may be omitted thus leaving us with only mass and momentum conservation equations. Equations are further simplified for incompressible flows. We can write those in Einstein notation as:

$$
\frac{\partial v_{j}}{\partial x_{j}}=0
$$

and

$$
\rho_{0} \frac{D v_{i}}{D t}=-\frac{\partial p}{\partial x_{i}}+\frac{\partial}{\partial x_{j}}\left(\nu \frac{\partial v_{i}}{\partial x_{j}}\right)+\rho_{0} g_{i},
$$

where $i$ is 1 or 2 for their planar forms, $v_{i}$ or $v_{j}$ are velocity vector components, $x_{j}$ are spatial coordinates, $g_{i}$ are gravity acceleration vector components, $t$ is time, $p$ is pressure and $\rho_{0}$ is density.

In equation (2), $\frac{D v_{i}}{D t}$ marks the material or Stokes derivative, which we can write in extended form as $\frac{D v_{i}}{D t}=\frac{\partial(\cdot)}{\partial t}+v_{j} \frac{\partial v_{i}}{x_{j}}$.

With some mathematical knowledge we can transform these equations to their velocity-vorticity form. By use of vorticity definition, the mass conservation equation can be transformed [3] to the following form: 


$$
\frac{\partial^{2} v_{i}}{\partial x_{j} \partial x_{j}}+e_{i j} \frac{\partial \omega}{\partial x_{j}}=0
$$

In equation (3) $\omega$ represents vorticity and $e_{i j}$ is unit permutation tensor. Since equation (3) does not have a unique solution we have to apply compatibility and restriction conditions for velocity and vorticity fields, thus obtaining the following equation for the kinematics of planar flows.

$$
\frac{\partial v_{i}}{\partial n}+e_{i j} \omega n_{j}=-e_{i j} \frac{\partial v_{j}}{\partial t},
$$

where $n_{j}$ are unit normal vector components. Momentum transport equations transform into the vorticity transport equation by applying the curl operator to equations (2)

$$
\frac{\partial \omega}{\partial t}+\frac{v_{j} \omega}{\partial x_{j}}=\nu_{0}\left(\frac{\partial^{2} \omega}{\partial x_{j} \partial x_{j}}\right)-\frac{1}{\rho_{0}} e_{i j} \frac{\partial f_{i}^{m}}{\partial x_{j}}
$$

where $f_{i}^{m}$ is the source term due to nonlinear viscosity and is equal to

$$
f_{i}^{m}=-e_{i j} \frac{\partial \widetilde{\eta} \omega}{\partial x_{j}}+2 e_{i j} \frac{\partial \widetilde{\eta}}{\partial x_{j}} \omega+2 \frac{\partial \eta}{\partial x_{j}} \frac{\partial v_{i}}{\partial x_{j}},
$$

where nonlinear dynamic viscosity is decomposed into $\eta=\eta_{0}+\widetilde{\eta}$.

Equation (5) is a scalar vorticity transport equation, since for planar flow, vorticity has only one component different from 0 .

For turbulence model Spalart-Allmaras [4] one equation low-Reynolds turbulence model was used, where the trip term was omitted.

$$
\begin{array}{r}
\frac{D \widetilde{\nu}}{D t}=C_{b 1}\left[1-f_{t 2}\right] \widetilde{S} \widetilde{\nu}+\frac{1}{\sigma}\left[\frac{\partial}{\partial x_{j}}(\right. \\
\left.\left.\left(\nu_{0}+\widetilde{\nu}\right) \frac{\partial \widetilde{\nu}}{\partial x_{j}}\right)+C_{b 2}\left(\frac{\partial \widetilde{\nu}}{\partial x_{j}}\right)^{2}\right] \\
-\left[C_{w 1} f_{w}-\frac{C_{b 1}}{\kappa^{2}} f_{t 2}\right]\left(\frac{\widetilde{\nu}}{d_{n}}\right)^{2},
\end{array}
$$

where $d_{n}$ is normal distance from the wall and $\widetilde{\nu}$ is a viscosity-like variable. Turbulent eddy viscosity is computed from:

$$
\nu_{t}=\widetilde{\nu} f_{v 1},
$$

where

$$
f_{v 1}=\frac{\chi^{3}}{\chi^{3}+C_{v 1}^{3}}
$$

and 
6 Advances in Fluid Mechanics VIII

$$
\chi=\frac{\widetilde{\nu}}{\nu_{0}}
$$

The rest of the functions are defined as:

$$
\begin{gathered}
f_{t 2}=C_{t 3} e^{-C_{t 4} \chi^{2}}, \\
f_{w}=g\left[\frac{1+C_{w 3}^{6}}{g^{6}+C_{w 3}^{6}}\right]^{\frac{1}{6}}, \\
g=r+C_{w 2}\left(r^{6}-r\right), \\
r=\frac{\widetilde{\nu}}{\widetilde{S} \kappa^{2} d_{n}^{2}}, \\
\widetilde{S}=S+\frac{\widetilde{\nu}}{\kappa^{2} d_{n}^{2}} f_{v 2}, \\
f_{v 2}=1-\frac{\chi}{1+\chi f_{v 1}}
\end{gathered}
$$

and

$$
S=\sqrt{2 \Omega_{i j} \Omega_{i j}}
$$

where $\Omega_{i j}$ is a rotation tensor.

The values of the constants are as shown in table (1).

Table 1: Constants of the Spalart-Allmaras turbulent model.

\begin{tabular}{|c|c|c|c|c|c|c|c|c|c|}
\hline$\sigma$ & $C_{b 1}$ & $C_{b 2}$ & $C_{w 1}$ & $C_{w 2}$ & $C_{w 3}$ & $C_{v 1}$ & $C_{t 3}$ & $C_{t 4}$ & $\kappa$ \\
\hline$\frac{2}{3}$ & 0.1355 & 0.622 & $\frac{C_{b 1}}{\kappa^{2}}+\frac{\left(1+C_{b 2}\right)}{\sigma}$ & 0.3 & 2 & 7.1 & 1.2 & 0.5 & 0.41 \\
\hline
\end{tabular}

\section{Integral equations}

Using $\xi$ as the collocation point, the integral equations of kinematics [5] take the form

$$
c(\xi) \vec{n}(\xi) \times \vec{v}(\xi)+\vec{n}(\xi) \times \int_{\Gamma} \vec{v} q^{*} d \Gamma=
$$




$$
\begin{array}{r}
\vec{n}(\xi) \times \int_{\Gamma}\left(\overrightarrow{q^{*}} \times \vec{n}\right) \times \vec{v} d \Gamma+\vec{n}(\xi) \times \int_{\Omega} \vec{\omega} \times \overrightarrow{q^{*}} d \Omega \\
c(\xi) \vec{n}(\xi) \cdot \vec{v}(\xi)+\vec{n}(\xi) \cdot \int_{\Gamma} \vec{v} \overrightarrow{q^{*}} d \Gamma= \\
\vec{n}(\xi) \cdot \int_{\Gamma}\left(\overrightarrow{q^{*}} \times \vec{n}\right) \times \vec{v} d \Gamma+\vec{n}(\xi) \times \int_{\Omega} \vec{\omega} \times \overrightarrow{q^{*}} d \Omega .
\end{array}
$$

$\Omega$ and $\Gamma$ denote domain and its boundary, $c$ is the geometric parameter depending on the location of source point and $q_{*}$ is the normal derivative of the fundamental solution.

Equations (18) and (19) are the tangential and normal forms of the kinematics integral equation, which we use for determining unknown boundary vorticity or tangential velocity component boundary values and unknown normal velocity component boundary values, respectively.

The integral equation of vorticity kinetics [3] is

$$
\begin{aligned}
c(\xi) \omega(\xi) & +\int_{\Gamma} \omega Q^{*} d \Gamma=\frac{1}{\eta_{0}} \int_{\Gamma}\left(\eta_{0} \frac{\partial \omega}{\partial n}-\rho_{0} v_{n} \omega+f_{t}^{m}\right) U^{*} d \Gamma \\
& +\frac{1}{\eta_{0}} \int_{\Omega}\left(\rho_{0} v_{j} \omega+e_{i j} f_{i}^{m}\right) Q_{j}^{*} d \Omega+\int_{\Omega} \omega_{F-1} u_{F-1}^{*} d \Omega .
\end{aligned}
$$

For weighting function of equations (18) and (19), the elliptic Laplace fundamental solution was used. Its planar form is:

$$
u^{*}=-\frac{1}{2 \pi} \ln (r),
$$

where $r$ is the distance between the source and the reference point. The normal derivative is written as $q^{*}=\frac{\partial u^{*}}{\partial n}$.

For equation (20) the parabolic diffusive fundamental solution was used, which is for planar flow in the form of:

$$
u^{*}=\frac{1}{4 \pi \alpha \tau}^{\frac{d}{2}} e^{\frac{-r^{2}}{4 \alpha \tau}},
$$

where $\alpha$ is the relaxation parameter, $d$ is the dimension of the problem and $\tau=$ $t_{F}-t$. Assuming constant variation of all field functions within the individual time frame $\Delta t$, the time integrals may be evaluated analytically;

$$
U^{*}=\alpha \int_{t_{F-1}}^{t_{F}} u^{*} d t
$$

and 


$$
Q^{*}=\alpha \int_{t_{F-1}}^{t_{F}} q^{*} d t
$$

The basic equation of the Spalart-Allmaras turbulent model (7) was partitioned to diffusive $\left(\nu_{0} \frac{\partial^{2} \widetilde{\nu}}{\partial x_{j}^{2}}\right)$, accumulation $\left(\frac{\partial \widetilde{\nu}}{\partial t}\right)$ and source term $(b)$ in order to obtain the corresponding integral equation;

$$
\begin{array}{r}
c(\xi) \widetilde{\nu}\left(\xi, t_{F}\right)+\int_{\Gamma} \widetilde{\nu} Q^{*} d \Gamma=\int_{\Gamma} \frac{\partial \widetilde{\nu}}{\partial n} U^{*} d \Gamma \\
+\frac{1}{\alpha} b U^{*} d \Omega+\int_{\Omega} \widetilde{\nu}_{F-1} u_{F-1}^{*} d \Omega
\end{array}
$$

\subsection{Numerical algorithm}

First, the laminar solution was obtained at some low Reynolds number [6]. Then calculation was restarted using the laminar results as initial conditions. The numerical algorithm calculates kinematics matrices first before entering the nonlinear loop. For laminar flows in the beginning of the nonlinear loop, the kinematics of flow is solved. The next step is obtaining a solution for vorticity transport, after that the loop begins anew. For turbulent flows, the algorithm first calculates turbulent viscosity by solving the turbulence model equation(s). After that, the nonlinear loop is the same as for laminar flows with the addition of calculating turbulent viscosity after the vorticity transport equation.

Kinematics is solved by a single-domain BEM. Kinetics is solved by a subdomain BEM that uses quadrilateral elements for discretization of the domain.

\section{Validation of the vorticity transport equation}

The nonlinear vorticity transport equation was validated by calculating flows with various viscosity distributions as shown in table 2 . Uniform meshes of different densities were used to test possibilities of capturing the nonlinearity of equations.

Table 2: Nonlinear dependence of viscosity for different test cases and values of the $v_{x}$ component of velocity at channel half width; mesh a) 60x6 elements, mesh b) 70x20 elements, mesh c) 80x40 elements.

\begin{tabular}{|c|c|c|c|c|c|}
\hline$\nu$ & $1+y$ & $1+100 \cdot y$ & $y^{3}$ & $y^{5}$ & $2-y$ \\
\hline Mesh a) & 0.08515 & 0.00406 & 0.03729 & 0.01541 & 0.08515 \\
Mesh b) & 0.08499 & 0.00362 & 0.03700 & 0.01511 & 0.08499 \\
Mesh c) & 0.08500 & 0.00356 & 0.03713 & 0.01504 & 0.08500 \\
Analytical & 0.08496 & 0.00352 & 0.03704 & 0.01509 & 0.08496 \\
\hline
\end{tabular}




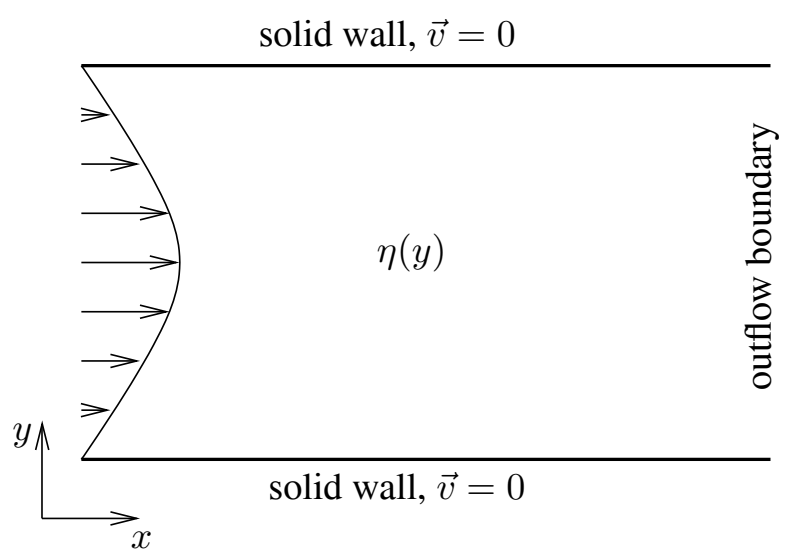

Figure 1: Boundary conditions for test cases.

The geometry for test cases was simple channel with a prescribed velocity profile at the inflow and zero flux boundary conditions at the outflow as shown in Figure 1.

The generalized equation in form $\frac{\partial}{\partial y}\left(\nu \frac{\partial v_{x}}{\partial y}\right)=-c$ was solved for different viscosity distributions and thus its behavior tested for nonlinear viscosity problems. First, viscosity was set to be the function of $1+k \cdot y$. If $0 \leq y \leq<1$, the general solution of this equation has the form:

$$
v_{x}(y)=\frac{c}{k}\left[-y+\frac{\ln (1+k y)}{\ln (1+k)}\right]
$$

Figures 2 and 3 show the results for two different values of $k ; 1$ and 100. For low values of $k$ governing equations are only weakly nonlinear, thus even coarse mesh describes the velocity profile quite well. At higher $k$ values coarser meshes are simply not good enough, hence the need for much finer meshes. Since gradients for the first two cases of viscosity distributions are constant, we prescribed viscosity distribution dependant on $y^{3}$ and $y^{5}$. If $1 \leq y \leq 2$ analytical solutions have forms of

$$
\begin{gathered}
v_{x}(y)=c\left[\frac{1}{y}-\frac{2}{3 y^{2}}-\frac{1}{3}\right], \\
v_{x}(y)=c\left[\frac{1}{3 y^{3}}+\frac{14}{45}\left(-\frac{1}{y^{4}}+1\right)-\frac{1}{3}\right] .
\end{gathered}
$$

A comparison of the analytical and numerical results is shown in Figure 4 for $y^{3}$ dependance and in Figure 5 for $y^{5}$ dependance of viscosity.

To test negative gradients, the viscosity was set to be function $\nu=2-y$. The analytical solution for the velocity profile is 

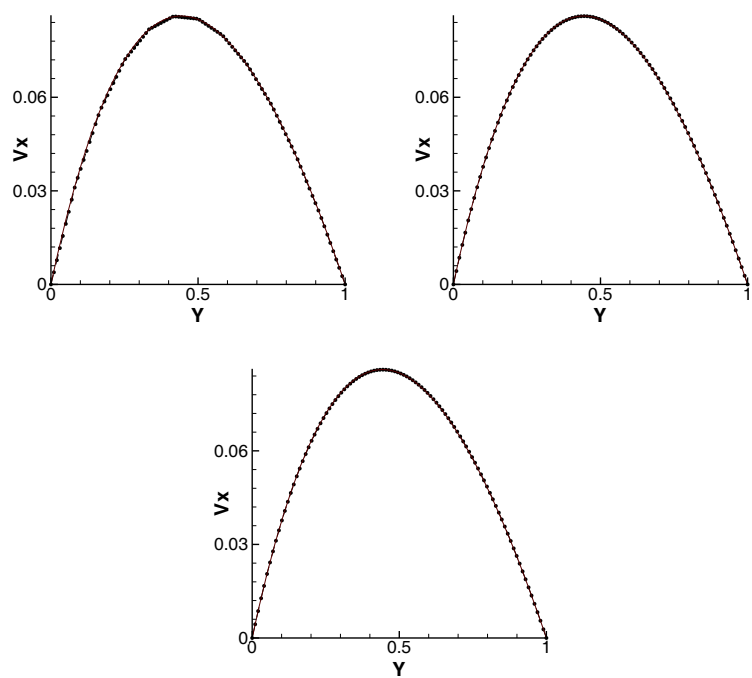

Figure 2: Velocity $\mathrm{x}$ component profiles for $\nu=1+y$ viscosity distribution; full circles represent numerical values, the full line represents analytical values; mesh a) top left, mesh b) top right and mesh c) bottom.
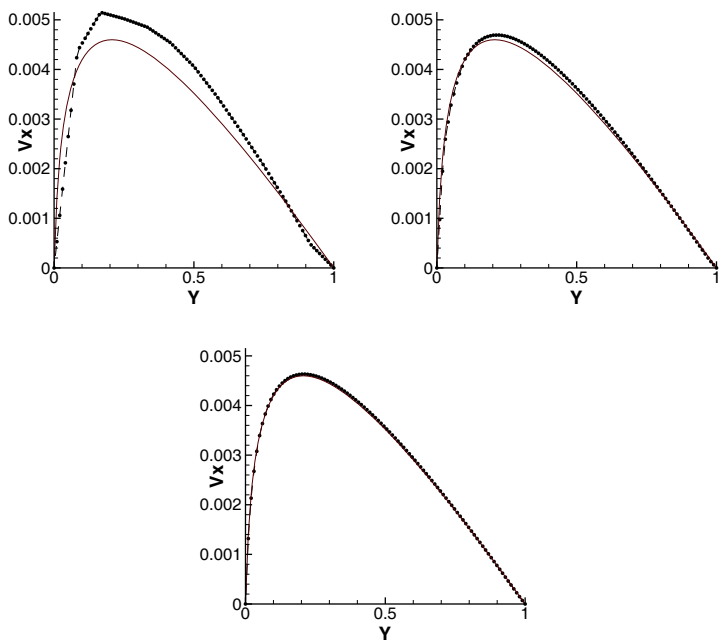

Figure 3: Velocity x component profiles for $\nu=1+100 \cdot y$ viscosity distribution.

$$
v_{x}(y)=c\left[1-y\left(\frac{\ln (2-y)}{\ln 2}\right)\right]
$$

while $y$ boundaries are the same as in cases of $\nu=1+k \cdot y$. The results for this test case are shown in Figure 6. 

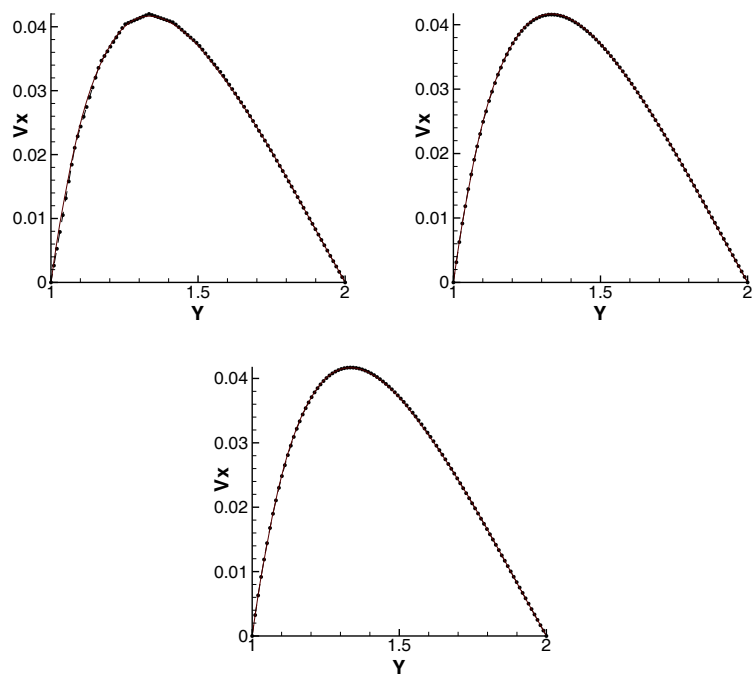

Figure 4: Velocity x component profiles for $\nu=y^{3}$ viscosity distribution.
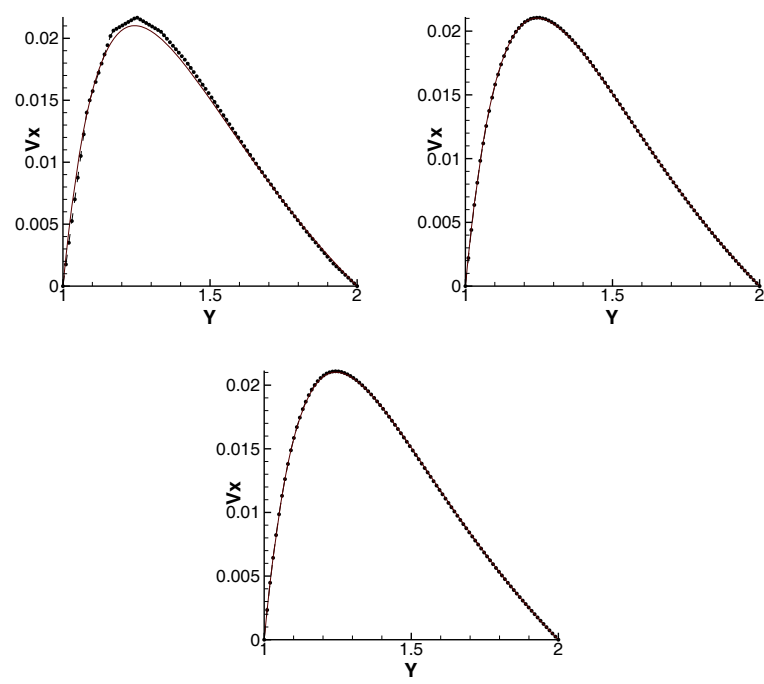

Figure 5: Velocity x component profiles for $\nu=y^{5}$ viscosity distribution.

\section{Conclusion}

The tested numerical algorithm was shown to be able to successfully cope with strong nonlinearities in the vorticity transport equation and it is able to predict the 

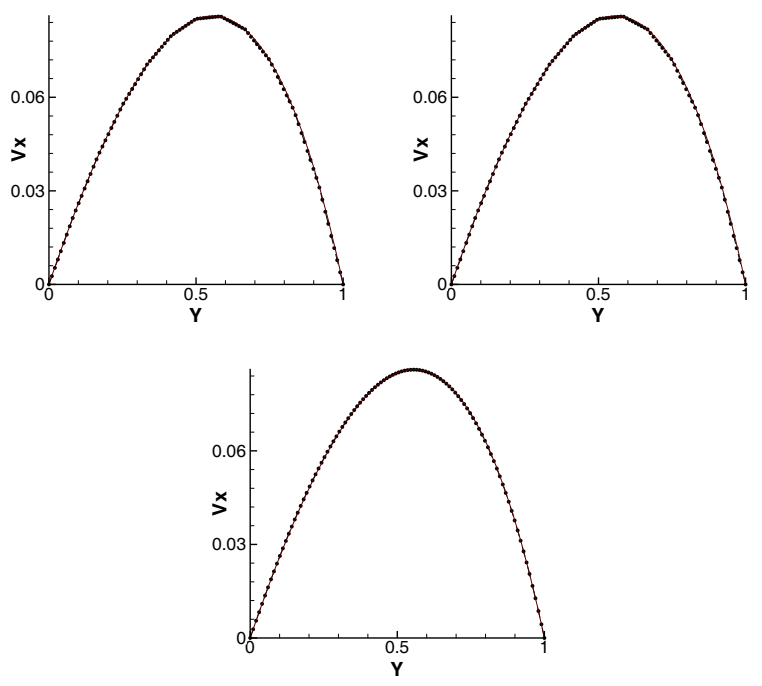

Figure 6: Velocity x component profiles for $\nu=2-y$ viscosity distribution.

corresponding velocity field accurately. In all test cases, except in the case with the strongest nonlinear viscosity distribution, even the coarsest mesh proved to be adequate. In the future, the developed numerical algorithm will be used to simulate turbulent fluid flow. DNS results of turbulent channel flow [7] will be used as a benchmark example to validate the algorithm. Furthermore, the algorithm will be tested on a backward-facing step example and compared with the DNS results of [8].

\section{References}

[1] Ravnik, J., Škerget, L. \& Hriberšek, M., The wavelet transform for BEM computational fluid dynamics. Eng Anal Bound Elem, 28, pp. 1303-1314, 2004.

[2] Paige, C.C. \& Saunders, M.A., LSQR: An algorithm for sparse linear equations and sparse least squares. ACM Transactions on Mathematical Software, 8, pp. 43-71, 1982.

[3] Škerget, L. \& Samec, N., BEM for the two-dimensional plane compressible fluid dynamics. Eng Anal Bound Elem, 29, pp. 41-57, 2005.

[4] Spalart, P. \& Allmaras, S., A one-equation turbulence model for aerodynamic flows. AIAA, Aerospace Sciences Meeting and Exhibit, 30 th, Reno, NV, p. 1992, 1992. 
[5] Škerget, L. \& Ravnik, J., BEM simulation of compressible fluid flow in an enclosure induced by thermoacoustic waves. Eng Anal Bound Elem, 33, pp. 561-571, 2009.

[6] Škerget, L., Hriberšek, M. \& Žunič, Z., Natural convection flows in complex cavities by BEM. Int J Num Meth Heat \& Fluid Fl, 13, pp. 720-735, 2003.

[7] Kim, J., Moin, P. \& Moser, R., Turbulence statistics in fully developed channel flow at low Reynolds number. J Fluid Mech, 177, pp. 133-166, 1987.

[8] Lee, H., Moin, P. \& Kim, J., Direct numerical simulation of turbulent flow over a backward-facing step. J Fluid Mech, pp. 330-349, 1997. 